\title{
Discrepant GH and IGF-I values in the evaluation of treated acromegalic patients; an ongoing challenge. A meta- analysis.
}

\section{G. Kanakis¹, A. Chrisoulidou², A. Bargiota ${ }^{3}$, Z.A. Efstathiadou ${ }^{4}$, L. Papanastasiou ${ }^{5}$, A. Theodoropoulou $^{6}$, S.K.Tigas ${ }^{7}$, D.A. Vassiliadi ${ }^{8}$, S. Tsagarakis ${ }^{9}$ and M. Alevizaki ${ }^{10}$}

1Department of Endocrinology, Athens Naval \& VA Hospital, Athens, Greece; ${ }^{2}$ Department of Endocrinology, Theagenio Cancer Hospital, Thessaloniki, Greece; ${ }^{3}$ Department of Endocrinology and Metabolic Diseases, University of Thessaly, Larissa, Greece; ${ }^{4}$ Department of Endocrinology, "Hippokration" General Hospital of Thessaloniki, Thessaloniki, Greece; ${ }^{5}$ Department of Endocrinology and Diabetes Center, Athens General Hospital "G. Gennimatas", Athens, Greece; 'Department of Internal Medicine, Division of Endocrinology, University Hospital of Patras, Rio, Greece; ${ }^{7}$ Department of Endocrinology, University of Ioannina, loannina, Greece; ${ }^{8}$ Endocrine Unit, 2nd Dept. of Internal Medicine - Propaedeutic, Research Institute and Diabetes Center, Attikon University Hospital, Medical School, National and Kapodistrian University of Athens, Greece; ${ }^{9}$ Department of Endocrinology, Evangelismos Hospital Athens, Greece; ${ }^{10}$ Endocrine Unit, Department of Medical Therapeutics, University of Athens, Medical School, Athens, Greece

\section{INTRODUCTION - OBJECTIVES}

Growth Hormone (GH) and Insulin-like Growth Factor 1 (IGF-I) are currently the principal biomarkers used to assess disease activity in acromegaly and any discrepancy between them, renders interpretation of results inconclusive. The purpose of this study was to assess the frequency of discrepant results and identify parameters that might affect the emergence of this phenomenon.

\section{METHODS}

A systematic review of Medline and Scopus was performed (1987 - 2013) followed by a meta-analysis to address the frequency of discrepant results between GH and IGF-I levels. Meta-regression was performed using the year of publication as a continuous variable and subgroup analyses included the different types of $\mathrm{GH}$ testing and $\mathrm{GH}$ assays used, as well as the results of patients treated with Somatostatin Analogues (SSAs) compared to those treated with non-pharmaceutical modalities.

\section{RESULTS}

The analysis retrieved 39 eligible studies totaling 7071 patients. The pooled discordance rate between $\mathrm{GH}$ and IGF-I was $25.7 \%$ (95\% Cl: 22.3-29.4) and the predominant format was that of elevated IGF-I with normal GH levels [15.3\% (95\% CI: 12.5-18.7)].
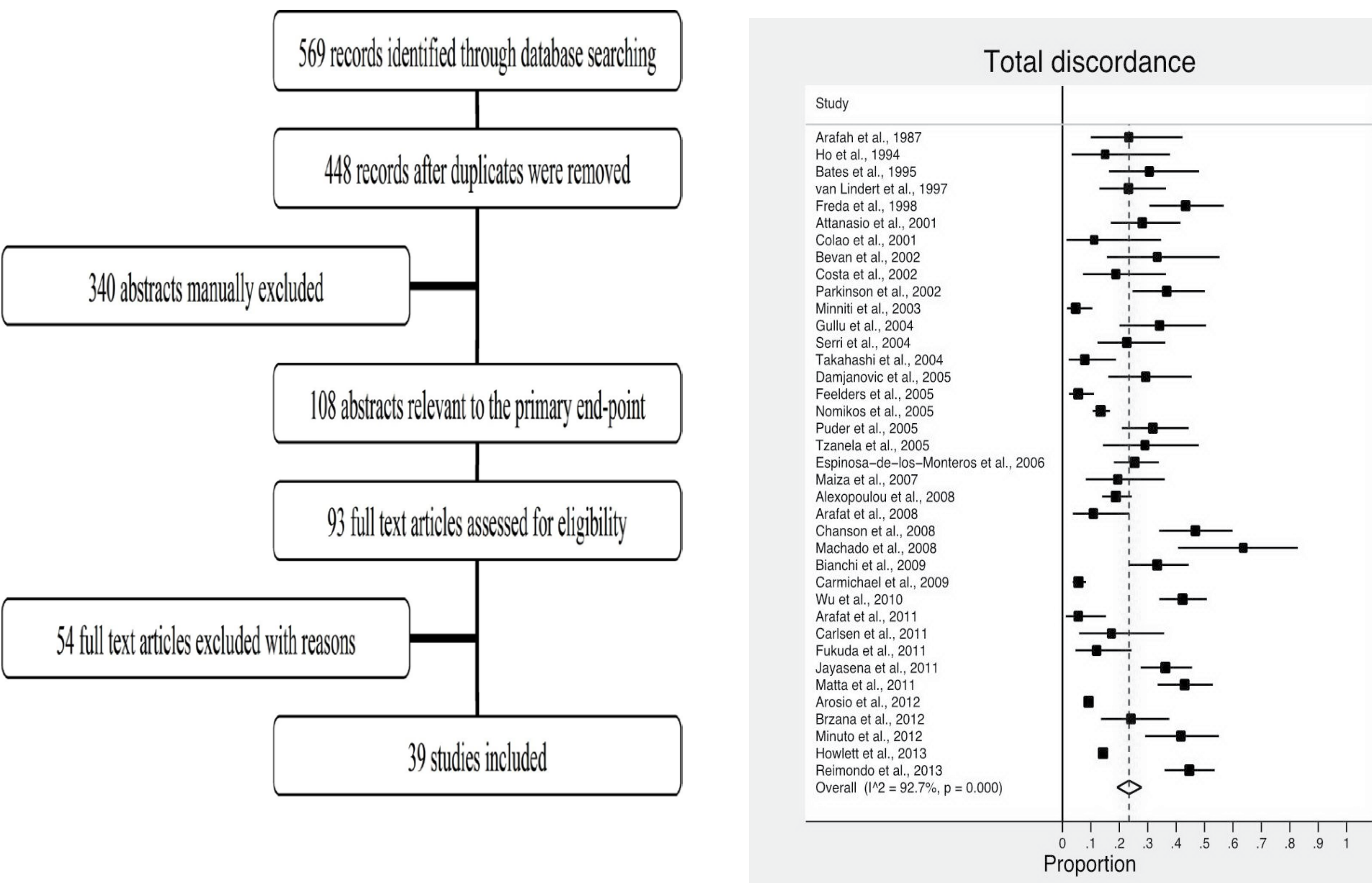

No significant correlation between the discordance rate and the year of publication was shown Coeff: +0.003 $95 \% \mathrm{Cl}:-0.008$ to +0.134 $(p=0.589)$
The use of ultrasensitive $\mathrm{GH}$ assays resulted in higher discordance rates $(30.7 \%, 95 \% \mathrm{Cl}$ : 25.9-35.9) vs. $(19.8 \%, 95 \% \mathrm{Cl}: 14.1-27.2, \mathrm{p}=0.04)$. On the contrary, $\mathrm{GH}$ nadir values obtained during an OGTT $(\mathrm{GHn})$ yielded the lowest discordance rates over a random $\mathrm{GH}$ value $(\mathrm{GHr})$ and over the mean value $(\mathrm{GHm})$ of a day curve $(22,0 \%$, vs. 29,1 vs. $33,5 \%$, $\mathrm{p}=0,041$ ).
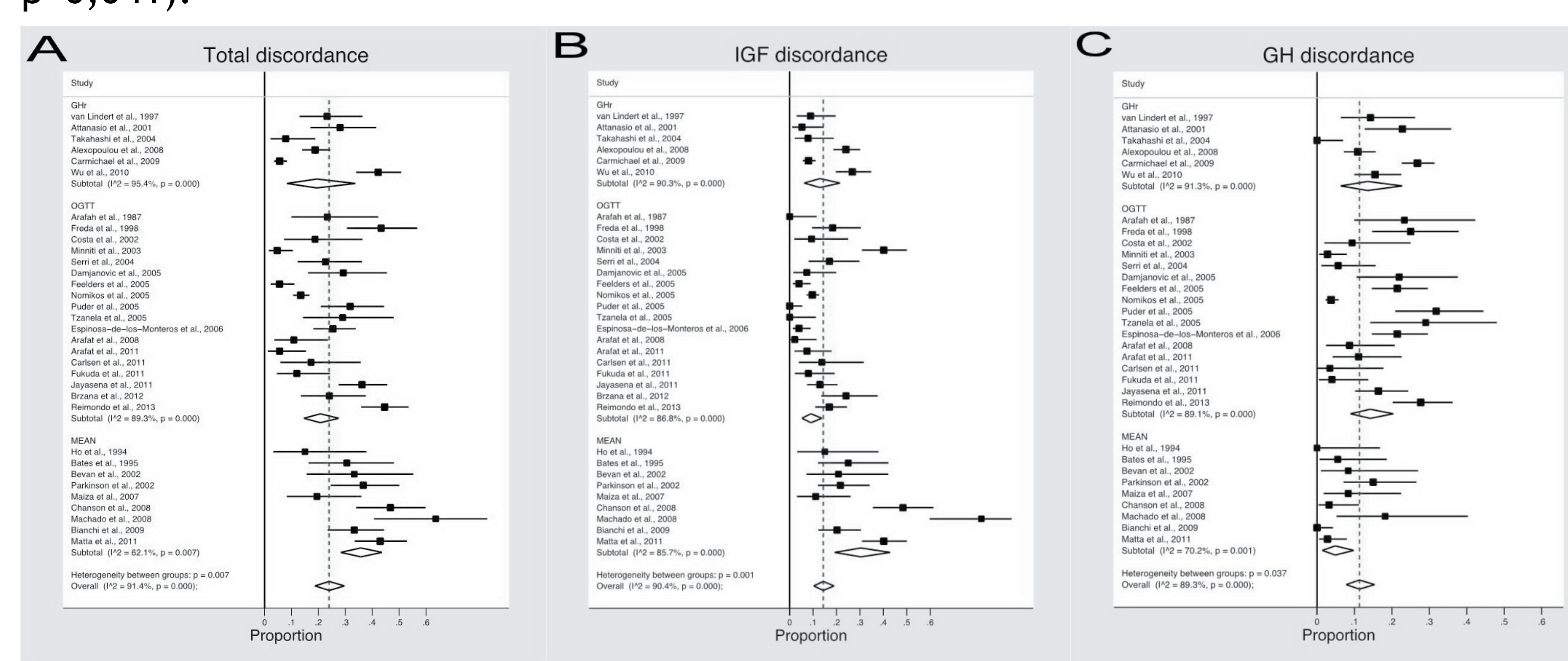

Patients receiving SSAs presented a discordance rate significantly higher than those not on pharmaceutical therapy $(32.5 \%, 95 \% \mathrm{Cl}: 27.8-37.4)$ vs. $(21.6 \%, 95 \% \mathrm{Cl}: 17.8-25.6$, $p=0.001)$
A

A

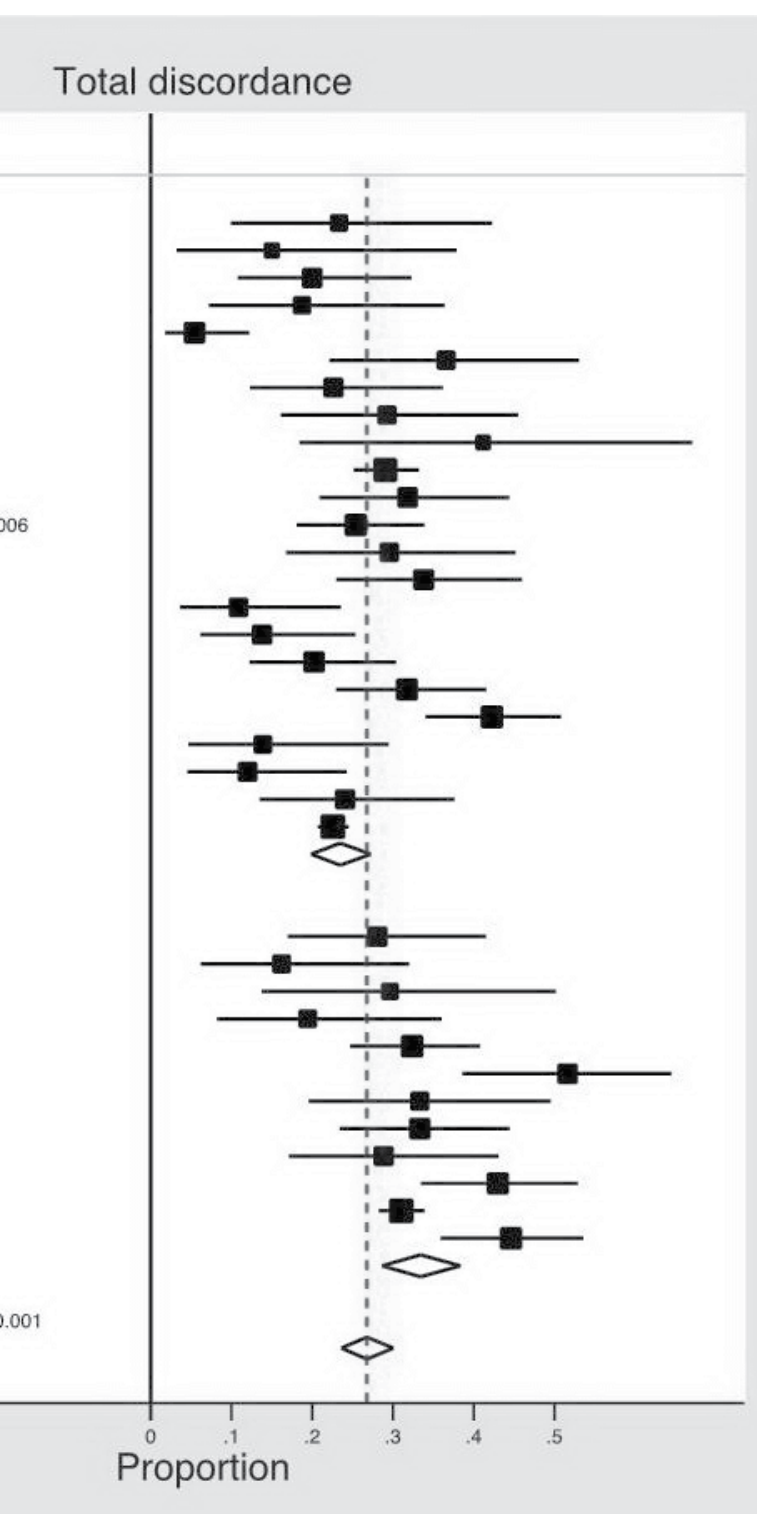

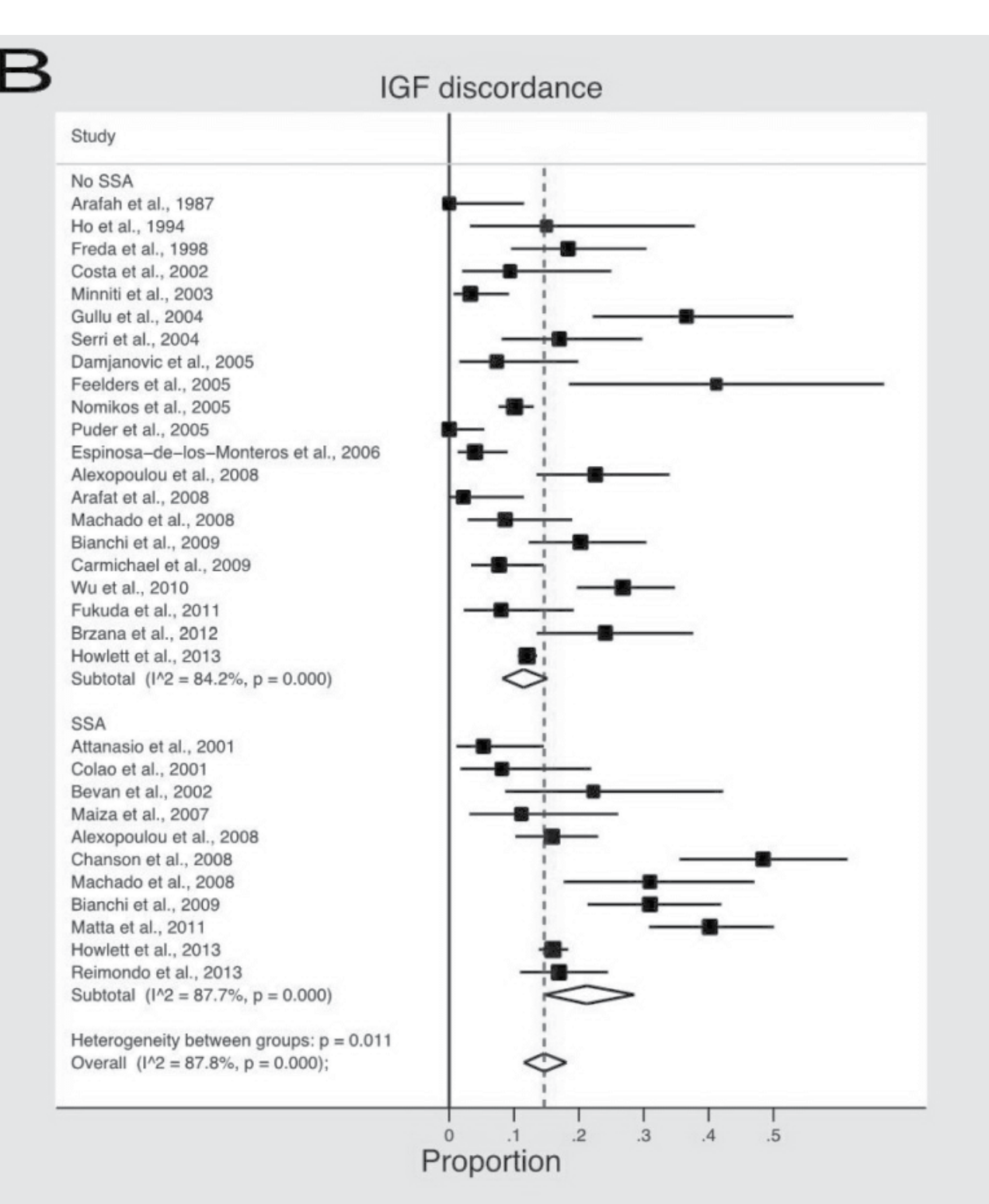

C GH discordance

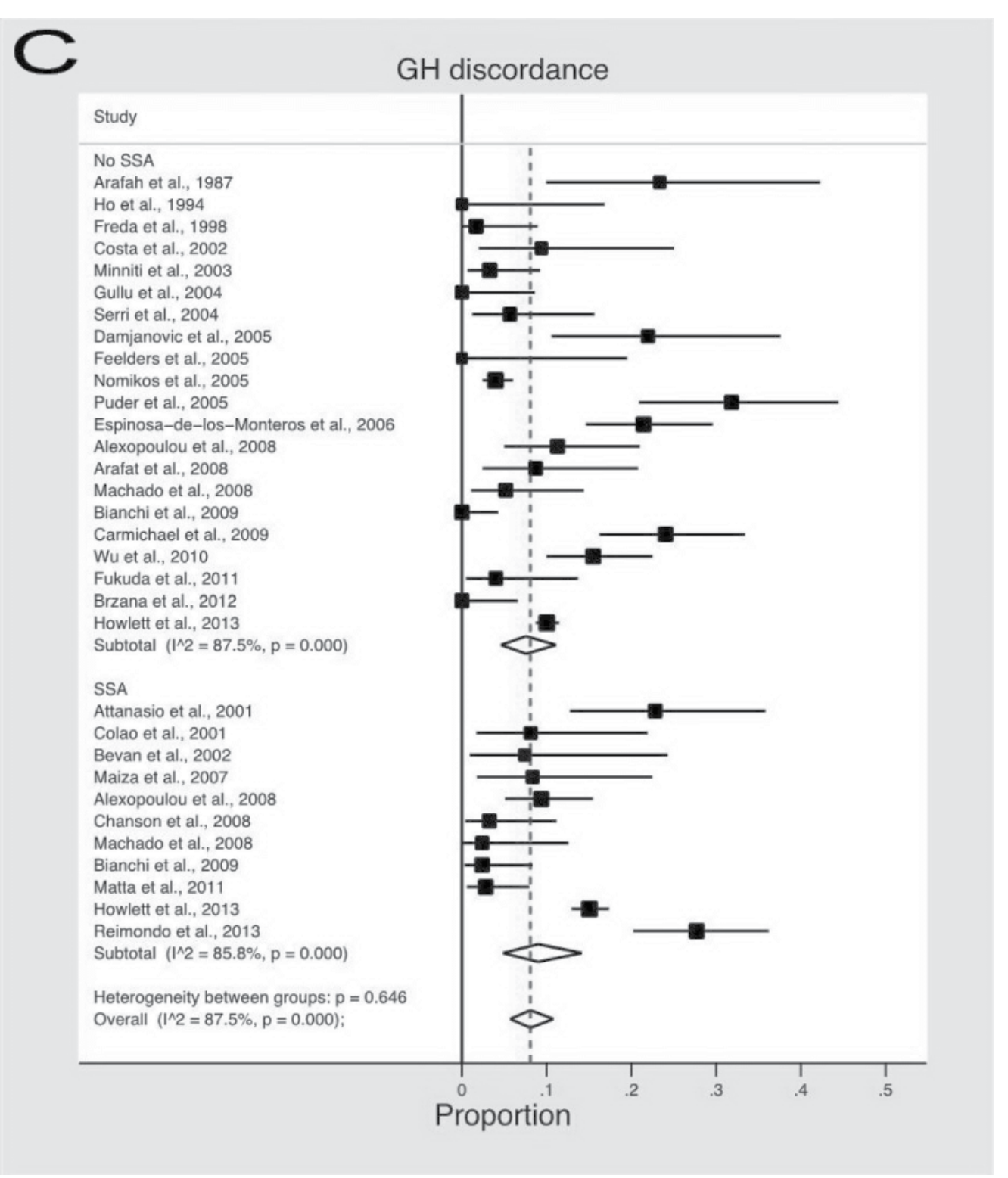

\section{CONCLUSIONS}

Discrepancy between GH and IGF-I results is encountered in almost a quarter of treated patients with acromegaly, especially when using ultrasensitive $\mathrm{GH}$ assays or in patients receiving SSAs, a fact that the clinician should take into consideration when making clinical decisions.

\section{REFERENCES}

1. Giustina, A Chanson, P., Bronstein, M. D., et al, (2010). A consensus on criteria for cure of acromegaly. J Clin Endocrinol Metab, 95 (7), 3141-8.

2. Alexopoulou, O., Bex, M., Abs, R., et al. (2008). Divergence between growth hormone and insulin like growth factor-i concentrations in the follow-up of acromegaly. J Clin Endocrinol Metab, 93 (4), 1324-30.

3. Freda, P. U. (2009). Monitoring of acromegaly: what should be performed when GH and IGF-1 levels are discrepant? Clin Endocrinol (Oxf), 71 (2), 166-70.

4. Zeinalizadeh, M., Habibi, Z., Fernandez-Miranda, J. C., et al. (2015). Discordance between growth hormone and insulin-like growth factor-1 after pituitary surgery for acromegaly: a stepwise approach and management. Pituitary, 18 (1), 48-59.

The Greek School of Endocrinology Initiative was organized and funded by Ipsen. Statistical support and publication costs associated with this work were also funded by Ipsen. The development at all stages of the concept and content was the sole responsibility of the authors.

\section{Lower Ribbon Requested by the Organizers}

